\title{
O uso da informação e a prática clínica de médicos residentes
}

\section{Maria Gorete Monteguti Savi}

\author{
Mestre em Ciência da Informação pela \\ Universidade Federal de Santa \\ Catarina.Bibliotecária da Universidade Federal de \\ Santa Catarina,
}

Edna Lucia da Silva

\begin{abstract}
Doutora em Ciência da Informação IBICTUFRJ.Professora do Departamento de Ciência da Informação e do Programa de Pós-Graduação em Ciência da Informação da Universidade Federal de Santa Catarina
\end{abstract}

Relato de pesquisa que aborda as necessidades informacionais e o uso de informação dos médicos residentes, na prática clínica. Contextualiza a pesquisa realizada no Hospital Universitário da UFSC, que teve como participantes os médicos residentes. Levanta as necessidades informacionais na prática clínica dos médicos residentes, que atuam nos ambulatórios do hospital. Mapeia o processo de escolha das fontes de informação e indica as mais utilizadas. Os resultados mostram que: durante sua prática ambulatorial, o médico residente se depara com questões classificadas como foreground, mais ligadas a aspectos clínicos do tratamento do paciente, em detrimento de questões ligadas a aspectos básicos, tais como epidemiologia e etiologia; utilizam como principais fontes de informação: periódicos, textbooks e handbooks, guidelines e preceptores, quase que de maneira equitativa; a Internet foi o meio mais utilizado na busca de informação; dentre os sistemas informatizados utilizados com frequência, destacam-se: UptoDate, Portal de Periódicos da CAPES e PubMed/Medline. Os resultados permitem inferir que os médicos residentes estão conscientes da importância do uso da informação para uma melhor prática clínica.

Palavras-chave: Uso da informação; Prática clínica; Médicos residentes; Hospital universitário. 


\section{Information use and clinical practice by resident physicians}

This paper describes the study of information requirements and use of information by resident physicians in clinical practice. It contextualizes the study undertaken at UFSC University Hospital of resident physicians and outlines the information requirements in clinical practice by the resident physicians in the hospital. It then maps the process of choosing the information sources to be used and shows those most widely used. The results suggest that: in the course of their work resident physicians are faced with issues classified as foreground issues which are more connected with the clinical aspects of patient treatment, to the detriment of issues connected with basic aspects such as epidemiology and etiology; for their main sources of information they use periodicals, textbooks and handbooks, guidelines and other manuals in an almost equal way; the internet was the most used method for information searches; within those information systems the most used were: UptoDate, the CAPES journal portal and PubMed/Medline. These results suggest that the resident physicians are aware of the importance of information use for more efficient clinical practice.

Keywords: Information Use; Clinical Practice; Resident Physicians; University Hospital.

Recebido em 08.06.2009 Aceito em 30.07.2011

\section{Introdução}

No cenário da sociedade da informação e de um mundo globalizado, no qual o desenvolvimento da ciência e tecnologia tem como consequência para a área médica a criação incessante de novos métodos diagnósticos, terapêuticos e preventivos, o acesso à informação para a prática clínica é essencial. Nesta pesquisa, procurou-se identificar as necessidades informacionais dos médicos residentes e as fontes de informação utilizadas no processo de busca de solução para as questões clínicas, surgidas no atendimento ambulatorial de um hospital.

Os resultados foram descritos e analisados a partir da coleta de dados obtidos através de questionário e entrevista aplicados aos médicos residentes do Hospital Universitário (HU) da UFSC. As informações obtidas nas entrevistas, realizadas através da técnica do incidente crítico, 
foram agrupadas por semelhança de conteúdo (categorias) e foram analisadas juntamente com as respostas dos questionários que apresentavam pertinência temática. Algumas questões elencaram respostas com indicação de prioridades, sendo que foi analisado até o nível de prioridade 3, por ter sido considerado o nível médio respondido nessas questões. Os dados transcritos farão indicação ao código alfanumérico da entrevista correspondente, compreendido entre E01 e E12. A análise e a interpretação dos dados realizaram-se privilegiando duas categorias: necessidades informacionais e fontes de informação.

Os resultados mais pontuais foram confrontados com pesquisas realizadas no Brasil e no exterior, a fim de se estabelecerem paralelos e de se identificarem semelhanças ou diferenças.

\section{Contextualização da pesquisa}

Para melhor entendimento das questões aqui tratadas, faz-se necessário a caracterização do lócus da pesquisa e seus dos participantes.

A pesquisa foi realizada com 38 médicos residentes, que atuam na prática clínica ambulatorial do Hospital Universitário Professor Polydoro Ernani de São Thiago da UFSC, sendo 22 mulheres (57,89\%), com idade mínima de 24 anos e máxima de 31 anos, e 16 homens (42,10\%), com idade mínima de 25 anos e máxima de 32 anos.

Com relação à distribuição dos pesquisados por ano, no Programa de Residência Médica, verificou-se que 42,11\% $(n=16)$ encontravam-se no 10 . ano $(R 1) ; 42,11 \%(n=16)$ no 20 . ano $(R 2)$; e $15,79 \%(n=6)$ no 30. ano (R3).Quanto aos anos de graduação acadêmica dos médicos residentes, verificou-se que estavam compreendidos no período de 1996 a 2006, destacando-se o ano de 2005, com 12 médicos residentes. Em relação à instituição em que se graduaram, observa-se que a UFSC formou a grande maioria dos médicos, 28 pesquisados $(73,68 \%) ; 6$ $(15,78 \%)$ eram provenientes de outras universidades do Estado de Santa Catarina: UNISUL e FURB, ambas com três $(7,89 \%)$; quatro eram provenientes de universidades brasileiras de outros estados: UNICAMP, UFMG, UFPel e UFRGS com um pesquisado para cada universidade $(2,63 \%)$.

Normalmente, as atividades dos médicos residentes ligados às áreas clínicas se dividem entre o atendimento ambulatorial, o atendimento nas unidades de internação, o atendimento na emergência e atividades teóricas. Os médicos residentes participantes desta pesquisa, indicaram que utilizavam a média de dez horas/dia (desvio padrão 1,77), com mínimo de oito horas/dia e máximo de 18 horas/dia, para suas atividades profissionais. Sendo que, para o atendimento ambulatorial, utilizavam a média de quatro horas/dia (desvio padrão 1,90), oscilando entre nenhum tempo e, no máximo, nove horas/dia (TAB. 1).

Nesta pesquisa, os dados referentes ao número de pacientes atendidos, diariamente, se ateve ao atendimento ambulatorial. Assim, com relação a 
essa variável, obteve-se a média de sete pacientes/dia (desvio-padrão 3,36), que oscilou entre zero e 16 pacientes/dia.

Tabela 1- Distribuição de horas dedicadas a RM, ao atendimento ambulatorial, às atividades teóricas e indicação do número de pacientes atendidos nos ambulatórios pelos médicos residentes do da UFSC

\begin{tabular}{l|c|c|c|c}
\hline \multicolumn{1}{c|}{ Medidas } & $\begin{array}{c}\text { Horas/dia dedicados } \\
\text { a RM }\end{array}$ & $\begin{array}{c}\text { Horas/dia de } \\
\text { atendimento } \\
\text { ambulatorial }\end{array}$ & $\begin{array}{c}\text { Horas/dia de } \\
\text { atividades teóricas }\end{array}$ & $\begin{array}{c}\text { Pacientes/dia } \\
\text { atendidos nos } \\
\text { ambulatórios }\end{array}$ \\
\hline Média & 10 & 4 & 2 & 7 \\
\hline Mínimo & 8 & 0 & 0,5 & 0 \\
\hline Máximo & 18 & 9 & 6 & 16 \\
\hline
\end{tabular}

Fonte: Dados da pesquisa.

\section{As necessidades informacionais na prática clínica}

Na prática clínica, o médico se depara com um número variável de questões que necessitam de respostas. Essas questões resultantes do fluxo informacional, que se estabelece na relação médico/paciente, e que geram uma necessidade de informação, deverão ser claras e objetivas, para que possam ser efetivamente respondidas. Para responder às questões clínicas, torna-se necessário que o médico busque informações para subsidiar esse processo, que deverão ser identificadas, interpretadas, integradas à realidade do paciente e aplicadas à decisão clínica.

Green, Ciampi e Ellis (2000, p. 220, tradução nossa) definiram "a necessidade informacional do médico como a exigência para novos conhecimentos médicos no que se refere ao cuidado de um paciente ou de um grupo de pacientes". Para Osheroff et al. (1991), as necessidades informacionais do médico são variáveis e complexas, e diversas fontes de informação são passíveis de consulta.

Outras variáveis também são apontadas na literatura, como fatores intervenientes nas necessidades informacionais. Para Leckie, Pettigrew e Sylvain (1996), os fatores demográficos, o contexto, a frequência, o grau de importância e a complexidade, podem direcionar as necessidades informacionais.

Na pesquisa realizada, foi identificado que os médicos residentes do HU da UFSC formulavam a média diária de 5,25 questões (desvio padrão 2,13 ), tendo sido indicado o mínimo de uma e o máximo de dez questões por dia. Considerando que a média de atendimento ambulatorial é de sete pacientes ao dia, conclui-se que os médicos residentes elaboravam 0,75 questão por paciente.

Alguns autores da literatura estrangeira apontam resultados aproximados. Dee e Blazek (1993) apontaram 0,3 necessidades por paciente; Gorman e Helfand (1995) e Green, Ciampi e Ellis (2000) 0,7; Cogdill et al. (2000) indicaram 0,29, quando o médico estava na presença de estudantes de medicina e 0,42, quando estava sozinho no atendimento clínico; Ramos, Linscheid e Schafer (2003) indicaram que entre os residentes o índice foi $1,5 \mathrm{e}$, entre os professores, de 0,8.

Procurou-se saber, também, qual o percentual de respostas encontradas para as questões clínicas formuladas. A média de respostas 
foi de $79 \%$ (desvio padrão de 15,20 ), com mínimo de $30 \%$ e máximo de $100 \%$ (TAB. 2). Portanto, temos a média de $21 \%$ de respostas não atendidas, ou seja, de cada cinco questões clínicas que foram realizadas diariamente, uma ficava sem resposta.

Resultado praticamente igual foi levantado por Green, Ciampi e Ellis (2000), que detectaram que os médicos residentes atenderam a $80 \%$ das questões. E, por Ramos, Linscheid e Schafer (2003), cujos resultados mostram que os médicos residentes atenderam a $74 \%$ e os professores a $39 \%$, ressaltando-se, nesta última pesquisa, o índice dos médicos residentes. Já na pesquisa de Covell, Uman e Manning (1985), os autores observaram um índice muito inferior, pois somente $30 \%$ das necessidades de informação dos médicos foram satisfeitas.

Com relação ao tempo gasto na resolução de cada questão clínica, foi indicado o tempo médio de 20 minutos (desvio padrão 15,46), com tempo mínimo de dois minutos e máximo de 60 minutos (TAB. 2). Na literatura estrangeira, verifica-se que existe diferença no tempo médio utilizado para resolução da questão clínica. Os dados da pesquisa de Woolf e Benson (1989) indicam que $49 \%$ dos médicos e estudantes de medicina levavam entre 10 e 30 minutos para a resolução de cada questão clínica. Os dados de Gorman (2001) indicam um tempo médio de 12 minutos; os de Green, Ciampi e Ellis (2000) a média é de 15 minutos; e na de Ramos, Linscheid e Schafer (2003) entre 5 e 15 minutos. Já contrastando com esse resultado, a pesquisa de Ely et al. (1999) indica um tempo muito inferior, ou seja, cada médico levava apenas dois minutos para responder a uma questão clínica. Essas três variáveis (número de questões clínicas, percentual de respostas encontradas e tempo gasto para resposta) foram calculadas com 37 pesquisados, já que um deles não informou esses dados.

Tabela 2- Indicação do número de questões clínicas formuladas, do percentual de respostas encontradas e do tempo gasto em cada resposta pelos médicos residentes do HU da UFSC

\begin{tabular}{l|c|c|c|c}
\hline \multicolumn{1}{c|}{ Variável } & Média & Mínima & Máxima & $\mathbf{N}^{\mathbf{0}}$ de respondentes \\
\hline Questões clínicas (indicação diária) & 5,25 & 01 & 10 & 37 \\
\hline Respostas encontradas (percentual) & $79 \%$ & $30 \%$ & $100 \%$ & 37 \\
\hline Tempo gasto em cada resposta (minutos) & 20 & 02 & 60 & 37 \\
\hline
\end{tabular}

Fonte: Dados da pesquisa

Com relação à frequência com que os médicos buscavam informação para resolver suas questões, a pesquisa apontou que $42,10 \% \quad(n=16)$ indicaram quase todos os dias, $31,58 \%(n=12)$ duas ou três vezes por semana, $23,68 \%(n=9)$ buscavam todos os dias, e 2,63\% $(n=1)$ menos de uma vez por semana. A opção "uma vez por semana" não foi indicada por nenhum dos pesquisados. 
A elaboração da questão clínica deve considerar o enfoque do problema, que pode estar relacionado a aspectos básicos, tais como causa, fisiopatologia; ou a aspectos clínicos, como diagnóstico, tratamento ou prognóstico. Nesta pesquisa, foi identificado que a maioria $(73,68 \%$, $\mathrm{n}=28$ ) dos médicos residentes buscava resposta para questões clínicas direcionadas para tratamento, seguidas de questões relacionadas com diagnóstico $(21,05 \%, n=8)$ e, por último, com prevenção $(5,26 \%, n=2)$. Questões relacionadas a prognóstico, etiologia, epidemiologia e outros não tiveram nenhuma indicação.

Este resultado em que o alto índice das questões está relacionado com a terapêutica também fica explicitado nas falas dos pesquisados:

[...] eu utilizo o critério da fonte de informação, aquilo que chega mais próximo possível [...] da melhor terapêutica que existe no momento (E01).

[São usados os] consensos pra ajudar na decisão terapêutica dos pacientes. (E05).

Também surgem nos depoimentos, questões relacionadas ao diagnóstico, segundo item indicado pelos médicos residentes do HU da UFSC, conforme as falas ilustrativas transcritas abaixo:

Pesquiso o que tem mais recente na literatura, em relação aos consensos na prática clínica, em relação àquela doença que estou suspeitando no paciente (E05).

Contribuíram pra diagnóstico. O diagnóstico estava difícil pra se fazer. Através de testes que eu aprendi nestes artigos, deu pra definir o diagnóstico do paciente (E07).

$\mathrm{Na}$ literatura nacional, a pesquisa de Martinez-Silveira (2005) aponta que as questões mais importantes estavam relacionadas ao diagnóstico, seguido do tratamento.

Alguns resultados presentes em literatura estrangeira corroboram com os resultados encontrados nesta pesquisa, quando indicam que as questões estão relacionadas com a terapêutica e o diagnóstico (DEE; BLAZEK, 1993; ELY et al., 1999; GREEN; CIMPI; ELLIS, 2000). Smith (1996), em trabalho de revisão que incluiu 13 pesquisas, concluiu que a maioria das questões clínicas estava relacionada com o tratamento do paciente.

Para os médicos residentes do $\mathrm{HU}$, a solução da questão clínica (GRÁF. 1) está frequentemente relacionada ao aumento do seu nível de conhecimento $(86,84 \%, \mathrm{n}=33)$, definição de intervenção no paciente $(63,16 \%, n=24)$, passar recomendações para o paciente $(28,95 \%, n=11)$, a modificar decisão anterior $(10,53 \%, n=4)$ e para programar retorno do paciente $(7,89 \%, \mathrm{n}=3)$. 
Gráfico 1 - Aplicabilidade da solução da questão clínica pelos médicos residentes do HU da UFSC

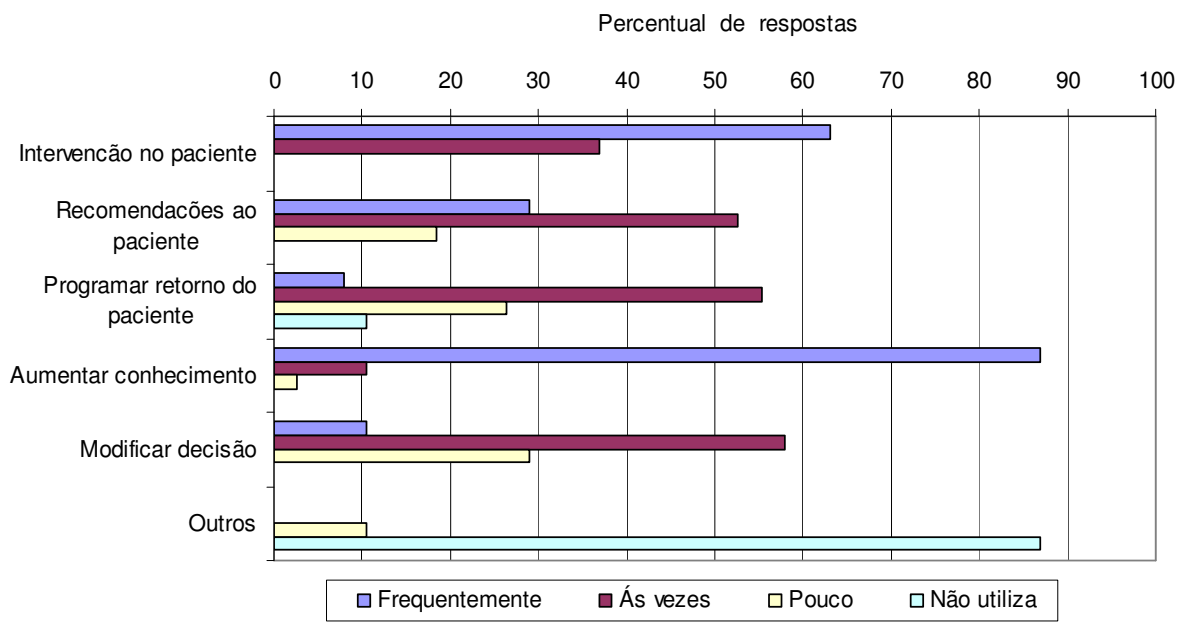

Fonte: Dados da pesquisa.

No item "às vezes", os resultados obtidos pelas opções de modificar decisão anterior, programar retorno do paciente, passar recomendações para o paciente e definição de intervenção no paciente ficaram mais equilibrados, entre 36,84\% e 57,89\% (entre 14 e 22 indicações). O alto índice $(86,84 \%, \mathrm{n}=33)$ da opção "outros" como não utilizados pelos médicos residentes indicou que todas as opções sobre a aplicabilidade da resposta da questão clínica foram sugeridas. As variáveis indicadas nas opções "pouco" e "não utiliza" foram, de maneira geral, inexpressivas.

Portanto, os médicos residentes do HU da UFSC elaboraram suas questões clínicas para problemas relacionados ao tratamento do paciente, seguido do diagnóstico. A solução das questões clínicas está frequentemente relacionada com o aumento do nível de conhecimento, ou seja, os médicos precisam de mais informações para melhor direcionar a prática clínica.

\section{A seleção de fontes e de canais de informação}

Atualmente, a grande quantidade de informação disponível na área médica tem exigido do médico o desenvolvimento de sua capacidade crítica para avaliar as fontes de informação disponíveis, a fim de selecionar as que possam responder mais adequadamente suas questões clínicas, seja através de canais formais ou informais, de fontes de informação impressas ou eletrônicas.

Para os médicos residentes do HU da UFSC que realizam atendimento ambulatorial, a informação é ponto de destaque na prática clínica, assumindo um papel primordial. A grande maioria, ou seja, 37 $(97,36 \%)$ dos médicos residentes, considerou que a informação científica é muito importante na prática clínica e apenas um deles $(2,63 \%)$ considerou "importante". As opções que indicavam "pouco importante" e "não considera importante" não tiveram nenhuma indicação. 
Os depoimentos, abaixo, reforçam a constatação de que a informação é muito importante para os médicos residentes, seja para esclarecer ou para decidir qual conduta médica tomar:

Contribuíram pra tomar a conduta, não necessariamente pra mudar uma decisão anterior (E10).

Contribui bastante. Porque a gente estava numa contradição, porque uma especialidade, no caso a [...] sugeria uma via de parto diferente da que a gente estava pensando pra esta paciente. E realmente no Cochrane a gente pode comprovar por metanálise que um número muito grande de pacientes com o mesmo problema igual à nossa [paciente], que a via de parto que a gente optou era a melhor pra aquela paciente. Foi bem legal, bem importante (E11).

De acordo com alguns depoimentos, percebe-se que a importância das fontes de informação está muito ligada à confirmação da resposta clínica, o que Smith (1996) indicou em sua pesquisa como a necessidade informacional que ultrapassa o conhecimento médico e se estende para uma sustentação psicológica, uma confirmação da resposta:

Acho que sim [importância da informação]. Principalmente, pra gente ter certeza daquilo que a gente está fazendo (E06).

Contribuíram bastante [as fontes de informação]. Só depois que a gente consultou [fontes de informação] é que foi tomada uma decisão. Antes disso, a gente estava bem reticente quanto a iniciar o tratamento, e foi depois desta consulta que foi começado (E08).

A questão referente aos principais meios que os médicos residentes utilizavam no processo de busca da informação (GRÁF. 2), para resolver suas questões clínicas, foi elucidada com base na indicação de prioridades. Com prioridade 1 (mais importante), a Internet foi apontada por $68,42 \%$ $(n=26)$, seguida da consulta ao acervo particular, com $13,16 \%(n=5)$ e da junção das opções: bibliotecas, contato com pares e contato com médicos da instituição, incluindo preceptores, com 18,41\% $(n=7)$. A opção "contato com médicos fora da instituição" não foi indicada com prioridade 1. 
Gráfico 2 - Busca da informação: canais de informação utilizados pelos médicos residentes do HU da UFSC

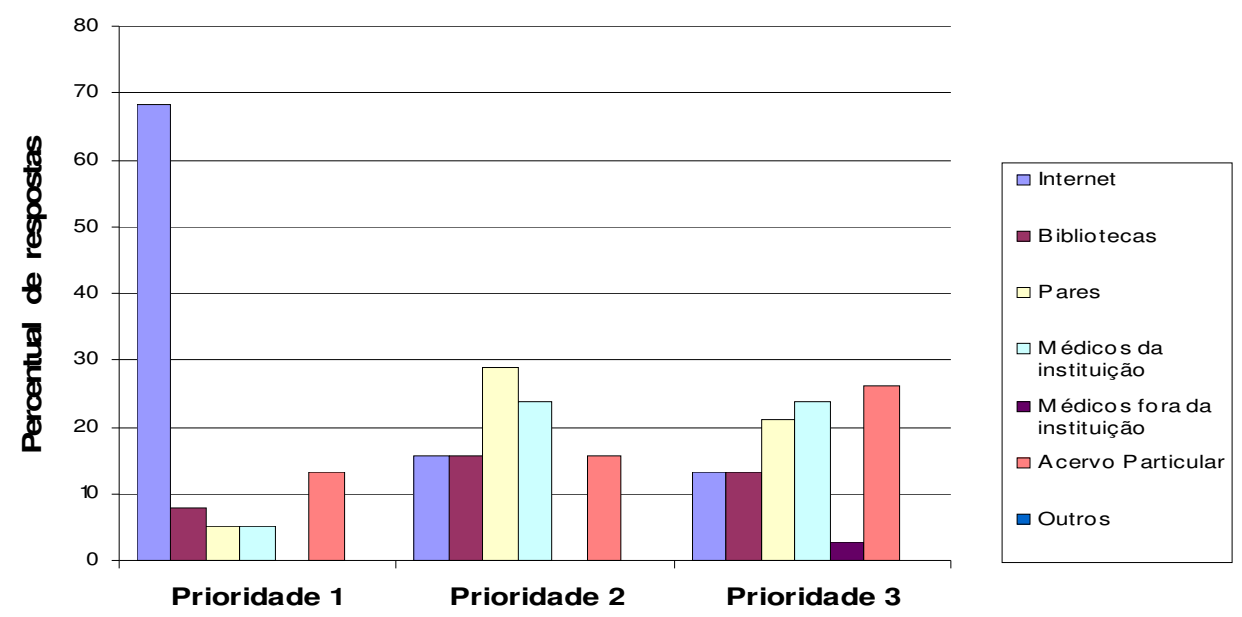

Fonte: Dados da pesquisa.

Este dado referente ao expressivo uso da Internet como canal de acesso à informação entre os profissionais da área da saúde já foi apontado em pesquisas mais recentes, que abordam exclusivamente esse tema, tais como a de Devitt e Murphy (2004), Cuenca e Tanaka (2005), Oliveira e Silva (2005), Renwick (2005) e Gómez-Tello et al.(2006).

Nas entrevistas realizadas com os médicos do HU, o uso da Internet como canal de informação é claramente evidenciado:

Quando eu precisei, a última vez que eu consultei foi a Internet (E04).

[...] mas na maioria das vezes [a consulta] foi na fonte on-line (E06).

Eu tenho procurado bastante em Internet, mas nos meios que eu considero confiáveis, sites de revistas: New England e Jama, ou então o UptoDate. Ou acabo consultando os colegas pela facilidade, afinal de contas está todo mundo junto e acaba discutindo as coisas (E08).

Porém, com relação à Internet, mesmo tendo sido considerada pelos médicos residentes do HU como a fonte mais utilizada, percebe-se que a questão da confiabilidade da informação encontrada nesse ambiente é apontada como fator preocupante pelos médicos residentes.

Com prioridade 2 prevaleceu o contato com os pares (outros médicos residentes) e com os médicos do próprio hospital, nos quais se enquadra a figura do preceptor. Eles foram os mais representativos, respectivamente com 28,94\% $(n=11)$ e $23,68 \%(n=9)$, conforme Gráfico 2.

Esse dado é compatível com os dados indicados na pesquisa de Haug (1997), que também apontou os pares como a opção mais indicada com prioridade 2 . 
As fontes de informação ajudam, nesse caso, a formar uma opinião clínica abalizada e permitem que a consulta e a discussão do caso, com profissionais mais experientes, sejam mais produtivas. Nas falas abaixo, essa afirmação é ratificada, já que os pesquisados inicialmente fazem menção ao uso da fonte de informação, para, só depois, indicarem a procura pelos preceptores e pares:

Bom, uma coisa que tenho utilizado bastante é o Uptodate [...] Também procurei, no caso, o staff e o residente mais graduado (E08).

A gente consulta guidelines, também a opinião dos chefes. E é basicamente isto. A gente consulta banco de dados [...] quando tem casos mais específicos tem que ir fazer a pesquisa (E09).

Em relação à consulta que os médicos residentes fazem aos pares, somente em um segundo momento, parece estar associado à responsabilidade que pode gerar para os pacientes uma decisão equivocada, já que, mesmo após a informação ter sido repassada por outro médico residente, o médico procura referendá-la na literatura. Abaixo, destacam-se algumas falas que ilustram essa situação:

[A informação] sugerida por colegas não era bem o que estava escrito nas fontes de informação (E01).

Colegas [consulto] mais pela facilidade. Tenho medo de pegar alguma coisa errada e trazer para a prática. Confiabilidade em primeiro lugar [...] Foi depois de olhar esta literatura um pouco mais que achei o artigo no Uptodate, mas foi um residente mais graduado que achou uma revisão, se não me engano foi no Chest, uma revista um pouco mais conceituada, e acabamos usando aquela informação e depois fui olhar no site para ver se a informação batia. Mas, é bem comum quando alguém toma uma decisão ou pra corroborar com o que a gente fez, dar uma olhadinha depois (E08).

No Brasil, a pesquisa de Breglia (1989) indica que os pares e outros médicos tiveram baixa expressão como fonte de informação, mas são muito procurados, especialmente os preceptores, como um guia para indicar qual a melhor fonte de informação a ser utilizada. Os dados da pesquisa de Martínez-Silveira (2005) apontam os preceptores e os pares como os mais consultados, seguidos da coleção particular; este último item coincide com esta pesquisa, que também indicou o acervo particular como o segundo mais importante meio de acesso às fontes de informação. $\mathrm{Na}$ pesquisa de Martínez-Silveira (2005), a Internet foi indicada como o terceiro canal de informação mais utilizado, enquanto que os médicos residentes do $\mathrm{HU}$ a apontaram como o mais utilizado.

$\mathrm{Na}$ literatura estrangeira, algumas pesquisas (STINSON; MUELLER, 1980; DEE; BLAZEK, 1993; RAMOS; LINSCHEID; SCHAFER, 2003) deixam 
claro que o contato com outro médico ${ }^{1}$ é indicado como o canal de informação mais importante, sendo que outras pesquisas, tais como Woolf e Benson (1989), Osherhoff et al. (1991), Cogdill et al. (2000) e Green, Ciampi e Ellis (2000) consideram como segunda opção mais importante.

Diferentemente desta pesquisa, a de Gorman (2001) identificou que os médicos que atendiam em comunidades rurais e urbanas pouco utilizavam os computadores e as bases de dados, preferindo contato com os pares.

Na prioridade 3, conforme mostra o GRÁF. 2, obtiveram indicações: consulta ao acervo particular com dez incidências (26,32\%), consulta aos médicos da instituição com nove $(23,68 \%)$, contato com colegas com oito $(21,05 \%)$, consulta à Internet e à biblioteca, ambas com cinco cada $(13,16 \%)$ e consulta a médicos de fora da instituição com uma $(2,63 \%)$.

O baixo índice de utilização da biblioteca indicado nesta pesquisa já foi percebido em pesquisas anteriores. Na pesquisa de Martínez-Silveira (2005), a biblioteca ocupou $06^{\circ}$. lugar na preferência dos médicos residentes. Na literatura estrangeira, Stinson e Mueller (1980) e Dee e Blazek (1993) também constataram a baixa utilização das bibliotecas.

Quando se depara com dificuldades para encontrar informações que possam responder sua questão clínica, ou seja, em um segundo momento do processo de busca de informação, o médico residente recorre a agentes que possam ajudá-lo. Na pesquisa, foram apontados como agentes desse processo (prioridade 1): preceptores $(63,15 \%, n=24)$, pares $(34,31 \%, n=13)$ e outros médicos da instituição $(2,63 \%, n=1)$, conforme GRÁF. 3 .

Com relação à importância do preceptor no processo de busca da informação, novamente ele aparece como mais importante, mas na hora da dificuldade. Em análise anterior, quando foram colocados em questão os meios utilizados na busca de informação, o preceptor não obteve dados expressivos dentre os indicados como prioridade 1, mas assumiu a primeira posição dentre os indicados na prioridade 2 .

Os bibliotecários só foram indicados na prioridade 3, com frequência de duas respostas $(5,26 \%)$. Portanto, novamente, obteve-se outro indicador que leva ao questionamento da posição da biblioteca e do bibliotecário nesse processo. A opção "outros" não foi indicada por nenhum dos pesquisados.

\footnotetext{
${ }^{1}$ Nas pesquisas indicadas, categorizou-se por "outro médico" os pares, os médicos da instituição e médicos de outras instituições.
} 
Gráfico 3 - Agentes de apoio informacional dos médicos residentes do HU da UFSC

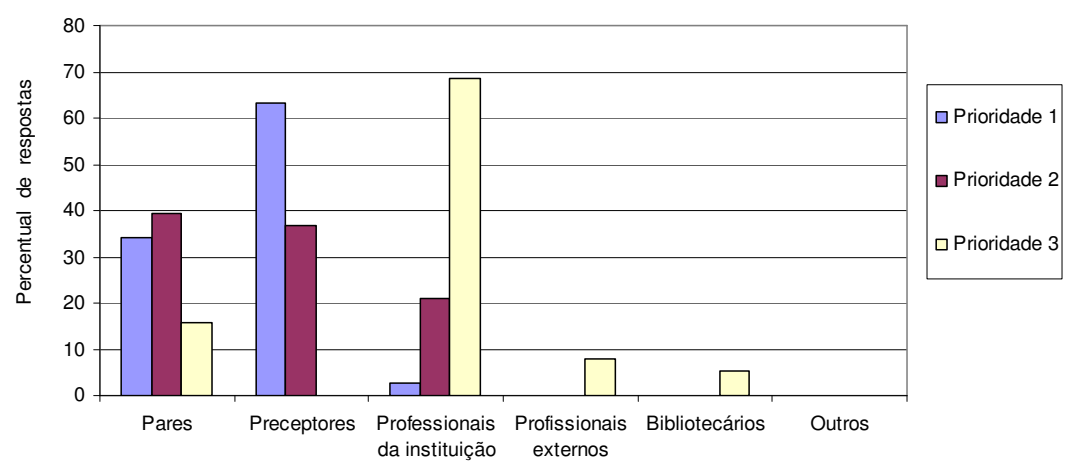

Fonte: Dados da pesquisa.

$\mathrm{Na}$ questão referente ao grau de frequência com que os médicos residentes utilizam as fontes de informação (GRÁF. 4), obteve-se nesta pesquisa, a indicação de uso frequente para os periódicos $(84,21 \%)$, textbooks e handbooks $(73,68 \%)$, guidelines $(73,68 \%)$ e preceptores $(73,68 \%)$ quase que de maneira eqüitativa, apenas com um pouco mais de ênfase nos periódicos.

Na prática clínica, as questões podem ser classificadas como básicas (background) e aplicadas (foreground). As respostas para questões básicas dependem de conhecimentos comumente relacionadas com etiologia, patogênese de doenças, mecanismos de ação e efeitos adversos de medicamentos. A questão aplicada (foreground) tem relação mais direta com a conduta a ser adotada, seja a conduta terapêutica ou conduta diagnóstica e, também, pode estar relacionada com 0 conhecimento sobre fatores indicadores de prognóstico. (BERNARDO; NOBRE; JATENE, 2003). De maneira geral, os dados da pesquisa apontam que, para os médicos residentes do HU da UFSC resolverem suas questões clínicas, são necessárias informações em nível de foreground em maior escala, geralmente disponíveis em periódicos técnico-científicos e guidelines e, em nível de background, geralmente disponíveis em textbooks (exceção para os baseados em evidência) e handbooks.

Sobre o uso frequente dos periódicos, constata-se que, ao longo do tempo, eles sempre assumiram uma posição de destaque na comunidade científica, porém, foram, paulatinamente, sendo incorporados no ambiente da prática profissional. Em relação ao uso dos periódicos no metiêr médico, a afirmação de Coutinho e Li (2004, p. 30) respalda o resultado desta pesquisa:

O processo de formação e transmissão do conhecimento sofreu uma mudança radical nas últimas décadas. Se, no passado, o tratado médico era a fonte de referência por excelência, hoje os periódicos científicos tomaram seu lugar, dada a grande rapidez na expansão do conhecimento. 
Os preceptores $(73,68 \%)$, pares $(60,53 \%)$ e outros especialistas $(50,00 \%)$ também são consultados frequentemente. Porém, quando se leva em consideração os dados analisados anteriormente, pode-se concluir que os mesmos são consultados com frequência, mas não em um primeiro momento.

Na literatura estrangeira, a pesquisa de Green, Ciampi e Ellis (2000) indicou que $28 \%$ das questões eram resolvidas com o uso de literatura de background e $66 \%$ de foreground.

De maneira geral, as mesmas fontes de informação indicadas na presente pesquisa aparecem como mais utilizadas na literatura revisada, porém, não necessariamente na mesma ordem.

Gráfico 4 - Fontes de informação: uso pelos médicos residentes do HU da UFSC

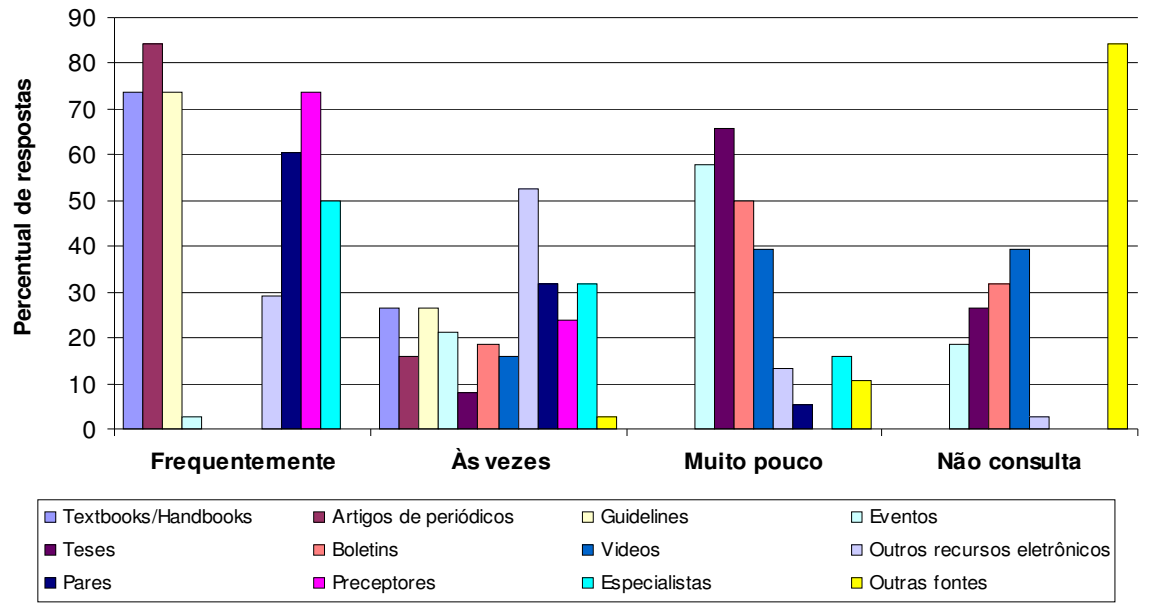

Fonte: Dados da pesquisa.

No Brasil, nas pesquisas de Martínez-Silveira (2005) e de Lima (2005), os livros aparecem como a fonte de informação mais consultada, seguidos dos periódicos. Porém, deve ser ressaltado que, em sua pesquisa, Lima (2005) colocou em uma única listagem as fontes de informação em seus diferentes suportes (por exemplo: CD-ROMs, slides, fitas de videocassete), juntamente com bases de dados específicas (por exemplo: Medline, Lilacs, Cochrane, SciELO), o que torna os resultados pouco reveladores. Breglia (1989) aponta que a fonte mais utilizada foram os textbooks, seguidos das bibliografias. Algumas pesquisas utilizam "livros" para designar os textbboks, handbooks, guidelines e outras publicações avulsas.

A revisão de 12 pesquisas realizadas por Haug (1997), entre o período de 1975 a e 1992, indica que os periódicos e os livros eram as fontes de informação mais consultadas. Coumou (2006), em revisão realizada no período de 1992 a 2005, indica os pares e as fontes de informação impressa. A pesquisa de Green, Ciampi e Ellis (2000) indica os textbooks, seguidos dos periódicos e dos preceptores. 
Na presente pesquisa, na opção "às vezes" são consultadas, foram indicadas as fontes de informação: recursos eletrônicos on-line, como imagens e sites institucionais com 52,63\%, pares e especialistas ambos com 31,58\%, e guidelines e textbooks/handbooks ambos com 26,32\%. Dentre as fontes indicadas como "muito pouco" consultadas, temos as teses $(65,79 \%)$, os trabalhos apresentados em eventos $(57,89 \%)$ e os boletins informativos (50\%). Entre as indicações de fontes não consultadas, temos outras fontes $(84,21 \%)$, os vídeos $(39,47 \%)$, os boletins informativos $(31,58 \%)$, as teses, dissertações e trabalhos acadêmicos $(26,32 \%)$, os trabalhos apresentados em eventos $(18,42 \%)$ e outros recursos eletrônicos on-line $(2,63 \%)$. O alto índice de indicação dos médicos residentes do HU para a opção "não consulta outras fontes de informação" (84,21\%), indica que as fontes de informação utilizadas, em maior ou menor escala, são as referendadas na questão. Os que responderam que utilizam outras fontes de informação com a opção "às vezes" $(2,63 \%)$ e com consulta "muito pouco" $(10,53 \%)$, não fizeram indicação de quais são essas outras fontes utilizadas.

Pelos dados desta pesquisa, em relação ao baixo índice de uso das teses, dissertações e trabalhos acadêmicos (indicação de "muito pouca" consulta - 65,79\%), pode-se inferir que não existe uma tradição de uso dessas fontes pelos médicos residentes do HU da UFSC. Esse mesmo resultado foi observado na pesquisa realizada por Lima (2005), na qual o uso das teses e dissertações impressas ocupou a $13^{a}$ colocação, e o uso das teses e dissertações eletrônicas a 16a. colocação. Na pesquisa de Breglia (1989), as teses e dissertações não foram incluídas entre as fontes consultadas em um primeiro momento; somente em um segundo momento elas aparecem, com apenas 0,9\%. Na literatura estrangeira analisada, essa variável não faz parte das abordagens realizadas.

O processo de busca da informação, por meio de bases de dados, tem sido alvo de muitas pesquisas, desde o início deste século (por exemplo: DEVITT; MURPHY, 2004; REWINCK, 2005, CUENCA; TANAKA, 2003; 2005 e GÓMEZ-TELLO et al., 2006), considerando o momento ímpar liderado pelo desenvolvimento das TIC e pela quantidade de informação técnico-científica produzida e disponibilizada diariamente. Para Bernardo, Nobre e Jatene (2004, p. 106), "as bases de dados informatizadas e acessadas pela Internet têm se constituído na principal fonte de busca de informação" para o médico.

A pesquisa com os médicos residentes do HU da UFSC sobre o uso de bases de dados (GRÁF. 5) indicou acesso frequente ao UptoDate $(71,05 \%)$, Portal de Periódicos da CAPES $(65,79 \%)$, PubMed - incluindo MEDLINE $(63,16 \%)$, bases de dados da BIREME $(39,47 \%)$, ferramentas de busca tipo Google, Cadê, Yahoo (26,32\%), SciELO (23,68\%), Cochrane Library $(21,05 \%)$, bases de dados Web of Science e EBMR (ambas com $5,26 \%$ cada) e MD Consult $(2,63 \%)$.

Gráfico 5 - Médicos residentes do HU da UFSC e o uso de sistemas informatizados de informação 


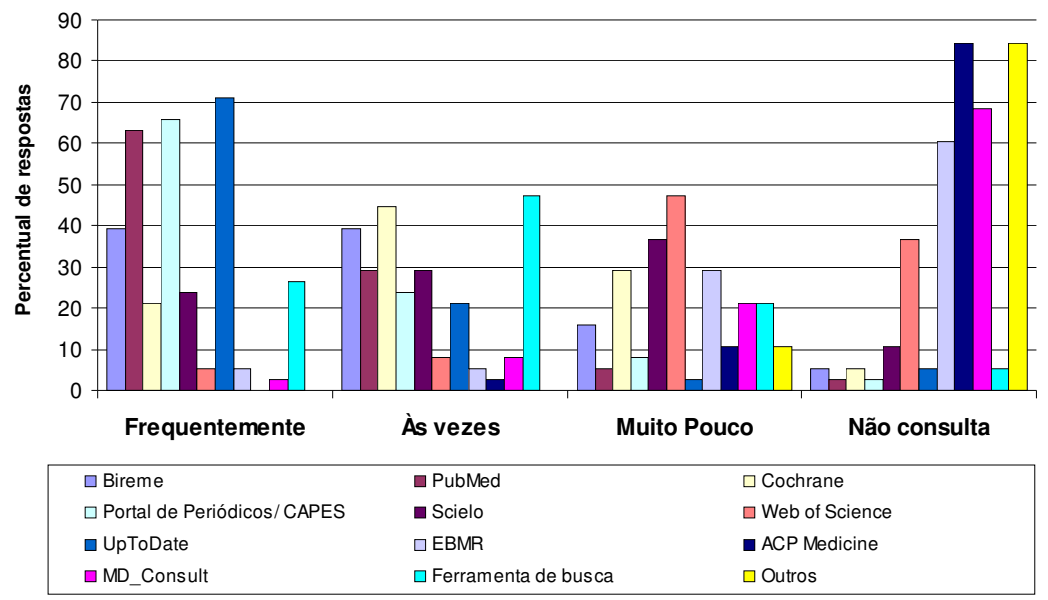

Fonte: Dados da pesquisa

Na entrevistas, o uso do Uptodate e PubMed foi referenciado como fontes de informação consultadas para resolver uma questão clínica relevante:

No PubMed apareceu vários artigos que me interessavam, selecionei os mais recentes e procurei fontes européias, porque já conhecia as americanas, para comparar as duas (E03).

[Consulto] na Internet, via PubMed. O que mais uso na minha prática clínica é usar o PubMed para ver o que tenho mais dúvida (E05).

Nas fontes que geralmente eu consulto [...] PubMed ou UptoDate e algumas coisas em livros (E06).

Bom, uma coisa que eu tenho utilizado bastante é o Uptodate, que o hospital assinou há pouco tempo. Primeiro por ser confiável e segundo por estar disponível facilmente em qualquer computador do hospital, e está disponível também no nosso quarto [quarto dos residentes], onde tem uma impressora e é muito fácil de imprimir. [...] UptoDate, que é um site idôneo que até hoje eu não vi comentário de erro nas revisões deles (E08).

Como a base de dados Uptodate, cujo conteúdo é conciso e dirigido especialmente para quem atua na prática clínica, foi assinada no ano de 2007 pela UFSC e disponibilizada nos computadores do $\mathrm{HU}$, deve ter desencadeado seu alto índice de uso. Na pesquisa de Ospina, Herault e Cardona (2005), foi revelado que os médicos acessavam cinco vezes mais as bases de dados gratuitas do que as que requerem pagamento. Além disso, outros diferenciais da base de dados Uptodate, que estão contidos em uma das falas citadas acima, referem-se à questão da confiabilidade da informação e à sua disponibilidade de acesso. 
Em relação ao PubMed, serviço criado pela NLM, que inclui as bases de dados MEDLINE, é importante ressaltar que o nome desse serviço, para a grande maioria dos médicos, é sinônimo da base de dados MEDLINE.

O alto índice de utilização do Portal de Periódicos da CAPES demonstra que esse instrumento está, cada vez mais, sendo incorporado no meio acadêmico e profissional.

As bases de dados com literatura nacional e latino-americana disponíveis no site da BIREME são utilizadas com certa cautela. As bases de dados da BIREME e a SciELO, mesmo que tenham obtido, respectivamente, a quarta e a sexta indicações de uso frequente, apresentaram uma grande diferença nos percentuais, quando comparadas com o uso do UptoDate e do PubMed. O depoimento de um entrevistado pode ser um indicador de motivo dessa diferença:

Ah! O Scielo que é outra fonte [consultada] e outra a Bireme, às vezes não tem artigos bons como na literatura inglesa ${ }^{2}$ (E03).

Na pesquisa de Martínez-Silveira (2005), a base de dados MEDLINE teve o mais alto índice de utilização $(71 \%)$, seguida pela LILACS e Cochrane Library, ambas com 12\%. Já na de Lima (2005), considerandose somente as bases de dados acessadas, tem-se: MEDLINE (ocupa a $3^{a}$. posição na listagem geral, que inclui também fontes impressas), LILACS (6a. posição), Cochrane (7a. posição), Outras bases da Bireme ( $8^{a}$. posição), SciELO (14a. posição), OVID (15a. posição) e ProBE (16a. última posição).

Entre os sistemas informatizados indicados como "às vezes" consultados, temos as ferramentas de busca $(47,37 \%)$, Cochrane Library $(44,74 \%)$, BIREME $(39,470 \%)$, PubMed e SciELO (ambos com $28,95 \%$ cada) e Portal de Periódicos da CAPES $(23,68 \%)$ e UptoDate $(21,05 \%)$. Percebe-se que as fontes de informação que foram indicadas como as mais utilizadas aparecem com os menores índices na opção "às vezes".

Um dado que surpreendeu foi a indicação de ferramentas de busca como a primeira opção em "às vezes" é consultada, além de constarem entre os usados "frequentemente", indicando que, mesmo utilizando fontes de informação de caráter científico, existia a procura de informação em mecanismos gerais de busca. Porém, considerando o que foi apontado anteriormente sobre a questão da confiabilidade dos sites, supõe-se que os médicos residentes, quando recorriam às ferramentas de busca, deviam empregar algum critério de seleção para uso das informações encontradas.

Dentre os sistemas indicados como "pouco consultados", os mais apontados foram Web of Science e SciELO, respectivamente com 47,37\% e $36,84 \%$.

Os sistemas de informação não consultados pelos médicos residentes do HU da UFSC tiveram alto índice para ACP Medicine e para outros sistemas (ambos com 84,21\%), sendo seguidos do MD Consult

\footnotetext{
${ }^{2}$ Nota dos autores: "literatura inglesa", referindo-se à literatura disponível em inglês.
} 
$(68,42 \%), \operatorname{EBMR}(60,53 \%)$ e Web of Science $(36,84 \%)$. Como as bases de dados ACP Medicine, MD Consult e EBMR não são bases de dados de acesso livre e a UFSC não mantém assinatura, este pode ser outro indicador de que a disponibilidade gratuita da informação é fator primordial na seleção das fontes de informação, conforme observado anteriormente.

Ospina, Herault e Cardona (2005), em pesquisa com médicos latinoamericanos dos países de língua espanhola, identificaram algumas fontes de informação mais consultadas, que são: MEDLINE, motores de busca geral (Google, Yahoo e Altavista) e BIREME.

O médico pode fazer uso da informação que foi selecionada e analisada antecipadamente, normalmente representada pelas fontes terciárias de informação, ou ele próprio realizar a seleção e análise da informação, através da consulta às fontes primárias ou secundárias. Na pesquisa, quando questionados sobre o tema, $26(70,27 \%)$ dos médicos residentes do $\mathrm{HU}$ da UFSC informaram que utilizavam a informação já selecionada e analisada, e $11(29,72 \%)$ preferiam realizar a seleção e análise da informação. Dessa forma, a maioria estava preferindo consultar fontes de informação terciárias, em detrimento do uso de publicações primárias e secundárias, que envolvem um processo mais complexo de seleção e análise da informação. Esse dado é confirmado na análise da questão anterior, quando se detectou que, dentre os sistemas informatizados de recuperação da informação, o UptoDate, base de dados que dispõe da informação já selecionada e analisada, apresentou o maior índice de utilização freqüente. Essa fonte foi complementada pelo PubMed que, dependendo dos critérios da estratégia de busca, pode, também, apresentar a informação já selecionada e analisada, também pelo acesso à Cochrane Library, que não teve forte indicação de uso frequente, mas que ocupou o segundo lugar na opção "às vezes".

Outro ponto enfocado na pesquisa está relacionado aos critérios que os médicos residentes utilizavam para escolher as fontes de informação. De acordo com o Gráfico 6, pode-se verificar que as respostas indicaram como muito importante a atualidade da informação com $84,21 \%(n=32)$, a confiabilidade da informação com $73,68 \%(n=28)$, a facilidade de acesso à informação com 71,05\% $(n=27)$, a facilidade de uso com $44,74 \%(n=17)$ e os custos da informação com 34,21\% $(n=13)$.

Pelos dados analisados nas questões anteriores, já foi possível perceber, especialmente pelas entrevistas, a incidência de três variáveis preponderantes nos critérios de seleção de fontes de informação, que agora são confirmadas como as mais importantes: atualidade, confiabilidade e acessibilidade.

$\mathrm{Na}$ opção "importante", por ordem de indicação obteve-se: custos da informação representando $47,37 \%(n=18)$, facilidade de uso com $44,74 \%$ $(n=17)$, confiabilidade com 23,68\% $(n=9)$, acessibilidade com $21,05 \%(n=8)$ e atualidade da informação com 15,79\% $(n=7)$. 
A maioria dos médicos residentes do HU da UFSC, exatamente 32 respondentes $(84,21 \%)$, indicou que não considera importante outros critérios além dos indicados na pesquisa.

Gráfico 6 - Critérios de seleção das fontes de informação dos médicos residentes HU/UFSC

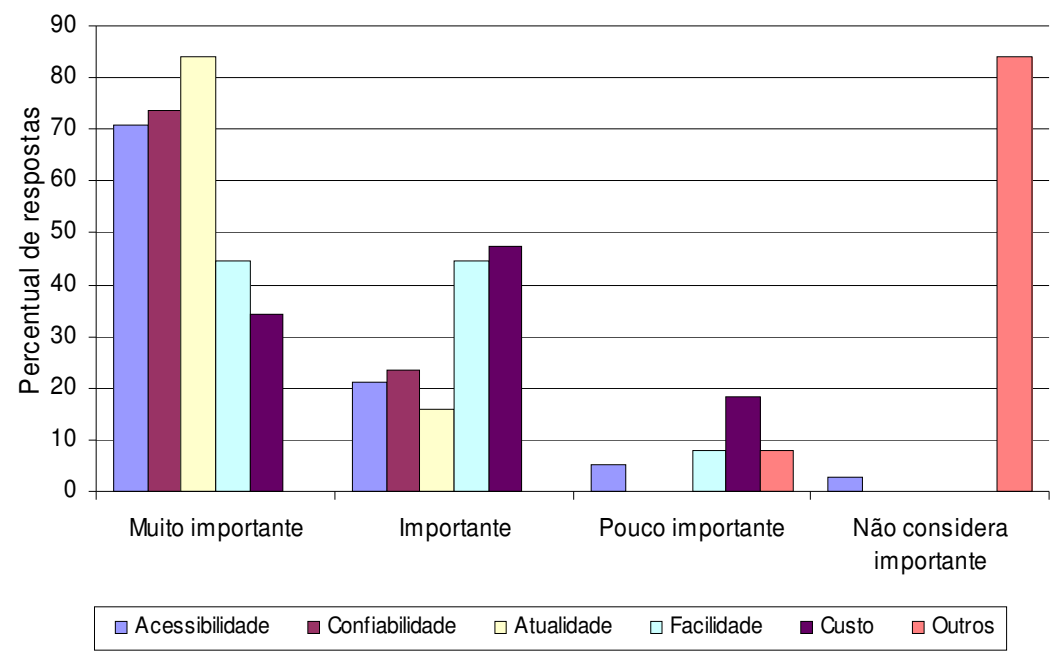

Fonte: Dados da pesquisa

Nos preceitos da metodologia da $M B E$, quando se busca na literatura, especialmente nos artigos de periódicos, respostas para as questões clínicas, o médico se depara com diferentes tipos de pesquisa ou desenhos de pesquisa e necessita determinar a sua validade e sua aplicabilidade para o problema em questão. Quando instigados sobre o tema, os médicos residentes do HU da UFSC indicaram como mais importantes os artigos de revisão com $44,74 \%(n=17)$, as revisões sistemáticas e/ou metanálises com $28,95 \%(n=11)$, os ensaios clínicos com 23,68\% $(n=9)$ e 2,63\% $(n=1)$ informou não ter preferências. Pelas entrevistas, percebe-se que os artigos de revisão e as metanálises predominam nas falas dos entrevistados:

Geralmente [o que consulto] são artigos de revisão. [...] Geralmente são revisões ou grandes metanálises (E04).

Dou preferência para os livros de revisão, metanálises. Teoricamente são melhores (E06).

[Utilizo] porque são artigos de revisão. Já selecionaram vários outros estudos, eles pegam os principais pra fazer os artigos de revisão. Por isto (E07).

[Utilizo] Portal que apresenta metanálise, [porque] estão uma seleção de vários trabalhos que passam por critérios rigorosos de metodologia, que foram selecionados pra montar uma fonte um pouco maior (E11).

O ensaio clínico, terceiro mais indicado pelos médicos residentes, teve seu uso ressaltado por um dos entrevistados: 
Geralmente eu utilizo o critério da fonte de informação, aquilo que chega mais próximo possível do ensaio clínico randomizado controlado duplo cego com placebo ou com a melhor terapêutica que existe no momento (E01).

A busca da informação na prática clínica interage com uma diversidade de motivos que impulsionam o médico a priorizar a busca de respostas.

Nesta pesquisa, verificou-se que os médicos residentes buscavam informação para resolver uma questão clínica, considerando como fator máximo de prioridade a urgência do caso clínico em 47,37\% $(n=18)$, o interesse pelo caso em $28,95 \%(n=11)$, dúvidas sobre os procedimentos no caso clínico em $19,53 \%(n=4)$, caso raro em $7,89 \%(n=3)$ e receio de não realizar uma boa prática e receio de cometer erro médico em $2,63 \%$ cada ( $\mathrm{n}=1$ cada), conforme indicado no GRÁF. 7 .

Gráfico 7 - Busca da informação: fatores priorizados pelos médicos residentes do HU da UFSC

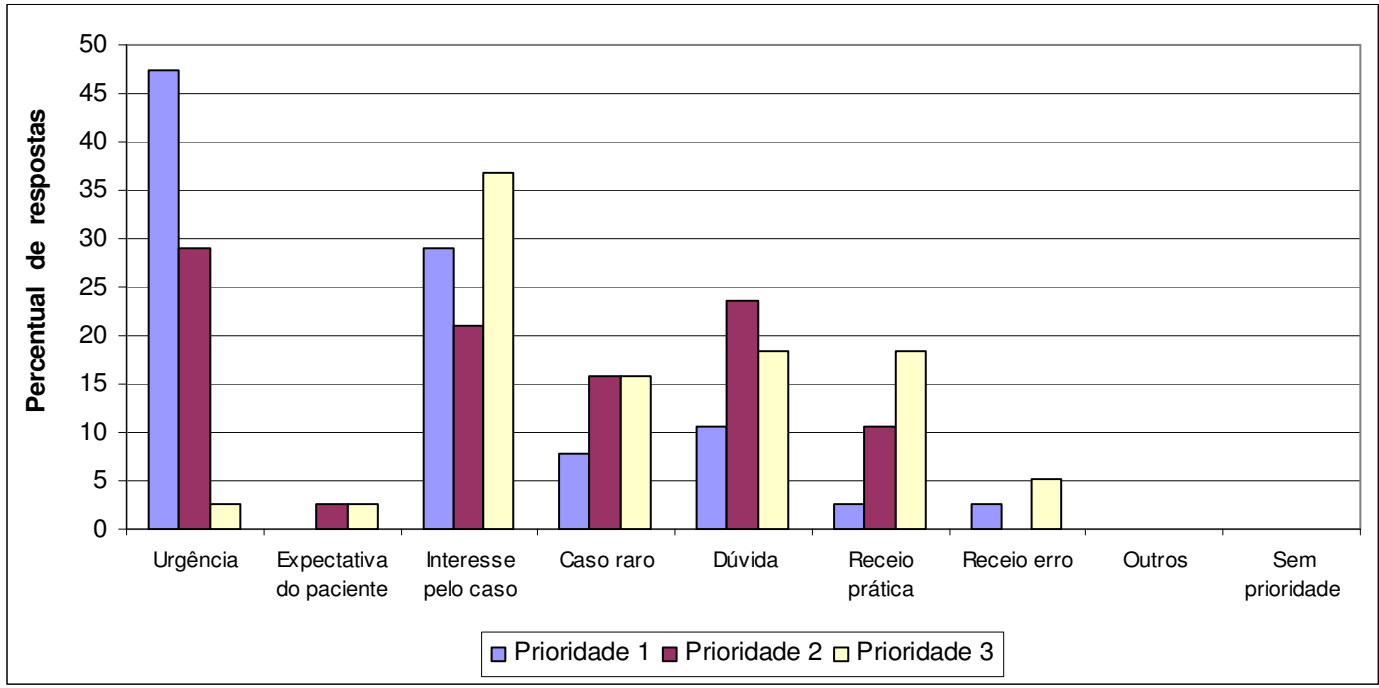

Fonte: Dados da pesquisa

Pesquisa anterior, realizada na Bahia por Martínez-Silveira (2005), aponta que a dúvida ou insegurança no caso clínico $(72,6 \%)$, a existência de caso raro $(71,2 \%)$, a apresentação do caso clínico em reunião $(67,1 \%)$ e o interesse pelo caso do paciente $(65,8 \%)$ eram os principais motivos que levavam os médicos residentes à busca de informação. Na literatura estrangeira, Green, Ciampi e Ellis (2000) constataram que os motivos estavam relacionados com a expectativa do paciente e o receio de cometer erro médico, este último item podendo ter estreita ligação com o rigor da legislação americana para casos dessa natureza. Na pesquisa de Gorman e Helfand (1995), foi identificado o mesmo resultado da presente pesquisa, ou seja, a urgência do caso clínico foi o fator que mais contribuiu para a busca da informação. 


\section{Considerações finais}

Notadamente, os médicos residentes do HU da UFSC estão conscientizados da importância da informação científica e já incorporaram o seu uso, como fator determinante do processo de decisão clínica. Dos resultados alcançados, foi possível perceber que, durante sua prática ambulatorial, o médico residente se depara com questões classificadas como foreground, mais ligadas a aspectos clínicos do tratamento do paciente, em detrimento de questões ligadas a aspectos básicos, tais como epidemiologia e etiologia. Tais resultados permitem inferir que 0 formando de medicina está saindo para o mercado de trabalho com um bom nível de conhecimentos, que suprem suas necessidades informacionais, relacionadas a aspectos básicos da profissão.

Dentre as fontes de informação utilizadas com mais frequência pelos médicos residentes do HU da UFSC, foram indicados os periódicos, com pequena vantagem sobre os textbooks e handbooks, os guidelines e os preceptores. Dentre as fontes com menor indicação de uso estavam os vídeos, os boletins informativos, as teses, dissertações e os trabalhos acadêmicos e os apresentados em eventos.

Com relação aos sistemas informatizados utilizados frequentemente, verificou-se a preferência pelo UptoDate, Portal de Periódicos da CAPES e PubMed/Medline. A preferência por informação já selecionada e analisada em idioma inglês, a confiabilidade da fonte de informação e a gratuidade no acesso foram, em maior ou menor grau, determinantes na indicação das bases de dados. As bases de dados nacionais, como as da BIREME e a SciELO, eram utilizadas com mais cautela.

O uso de fontes terciárias teve predominância, através da indicação de uso frequente da base de dados UptoDate, sendo que também tiveram destaque as fontes primárias, através do Portal de Periódicos da CAPES, e as fontes secundárias, com a indicação do PubMed.

Com relação ao uso das bases de dados, verificou-se que o médico residente do HU da UFSC dava preferência à busca por termos livres e estava deixando de usar os recursos disponíveis para tornar a recuperação da informação mais produtiva, em termos de relevância e pertinência. Essa constatação abre espaço para as bibliotecas oferecerem treinamento a usuários para uso das bases de dados, especialmente para explorar as potencialidades que cada sistema oferece.

Da análise empreendida, mesmo não sendo o foco principal desta pesquisa, vale destacar a pouca relevância dada à biblioteca nesse processo. Os resultados mostram que parece haver um importante papel a ser desempenhado pelo bibliotecário que atua na área médica, de modo que o planejamento dos serviços da biblioteca seja estruturado a partir de estudos de usuários que enfoquem as necessidade informacionais e as dificuldades encontradas pelo usuário na busca e recuperação da informação. Para tal, faz-se necessário investir no desenvolvimento de habilidades e de conhecimentos específicos do bibliotecário para essa atuação. 


\section{Referências}

BERNARDO, W. M.; NOBRE, M. R. C.; JATENE, F. B. A prática clínica baseada em evidências: parte II - buscando as evidências em fontes de informação. Revista da Associação Médica Brasileira, Rio de Janeiro, v. 50, n. 1, p.104-108, jan./mar. 2004. Disponível em: <http://www.sci.br/pdf/ramb/v50n1/ a45v50n1.pdf>. Acesso em: 20 jul. 2006.

BREGLIA, V. L. A. A comunicação da informação na residência médica. 1989. 203 f. Dissertação (Mestrado em Ciência da Informação)IBICT/Universidade Federal do Rio de Janeiro, Rio de Janeiro, 1989.

COGDILL, K. W. et al. Information needs and information seeking in community medical education. Academic Medicine, v. 75, n. 5, p. 484486, 2000.

COUMOU, H. C. H. How do primary care physicians seek answers to clinical questions? A literature review. Journal of the Medical Library Association, Chicago, v. 94, n. 1, p. 55-60, jan. 2006.

COUTINHO, M.; LI, S. M. Como ter acesso à literatura médica. In: DRUMMOND, J. P.; SILVA, E.; COUTINHO, M. Medicina baseada em evidências: novo paradigma assistencial e pedagógico. 2. ed. São Paulo: Atheneu, 2004. cap. 2.

COVELL, D. G.; UMAN, G. C.; MANNING, P. R. Information needs in office practice: are they being met? Annals of Internal Medicine, Philadelphia, $v$. 103, n. 4, p.596-599, Oct. 1985.

CUENCA, A. M. B.; TANAKA, A. C. D. As novas tecnologias na comunicação científica: o uso da Internet pela comunidade de docentes da área de saúde pública. In: ENCONTRO NACIONAL DE PESQUISA EM CIÊNCIA DA INFORMAÇÃO, 5., 2003, Belo Horizonte. Anais... Belo Horizonte: UFMG, 2003.

Influência da internet na comunidade acadêmico-científica da área de saúde pública. Revista de Saúde Pública, São Paulo, v. 39, n. 5, p. 840-846, 2005.

DEE, C.; BLAZEK, R. Information needs of the rural physician: a descriptive study. Bulletin of the Medical Library Association, Chicago, v. 81, n. 3, p. 259-264, 1993.

DEVITT, N.; MURPHY, J. A survey of the information management and technology training needs of doctors in an acute NHS trust in the United Kingdom. Health Information and Libraries Journal, v. 21, n. 3, p. 164172, 2004.

ELY, J. W. et al. Analysis of questions asked by family doctors regarding patient care. British Medical of Journal, London, v.319, p. 358-361, Aug. 1999. Disponível em: <http://www.bmj.com/cgi/reprint/319/7206/358>. Acesso em: 27 mar. 2007. 
GOMÉZ-TELLO, V. et al. Uso de Internet y recursos electrónicos entre médicos intensivistas españoles: primera encuesta nacional. Medicina Intensiva, Madrid, v. 30, n. 6, Aug./Sept. 2006.

GORMAN, P. N. Information needs in primary care: a survey of rural and nonrural primary care physicians. Medinfo, Edmonton, v. 10, pt. 1, p. 338-342, 2001.

GORMAN, P. N.; HELFAND, M. Information seeking in primary care: how physicians choose which clinical questions to pursue and which to leave unanswered. Medical Decision in Making, v. 15, n. 2, p.113-119, 1995.

GREEN, M. L. ; CIAMPI, M. A.; ELLIS, P. J. Resident's medical information needs in clinic; are they being met? American Journal of Medicine, Newton, v. 109, p. 218-223, Aug. 2000.

HAUG, J. D. Physicians' preferences for information sources: a metaanalytic study. Bulletin of Medical Library Association, Chicago, v.85, n.3, p.223-232, July $1997 . \quad$ Disponível em: http://www.pubmedcentral.nih.gov/picrender.fcgi?artid=226263\&blobtype =pdf $>$. Acesso em: 26 mar. 2007.

LECKIE, G. J..; PETTIGREW, K. E.; SYLVAIN, C. Modeling the information seeking of professional: a general model derived from research on engineers, health care professionals and lawyers. Library Quartely, v. 66, n. 2, p. 161-193, 1996.

LIMA, H. M. C. Experiências em buscas de informações por residentes de medicina. Revista Brasileira de Educação Médica, Rio de Janeiro, v. 29, n. 1, p. 13-21, jan./abr. 2005.

MARTINEZ-SILVEIRA, M. S. A informação científica na prática médica: estudo do comportamento informacional do médico residente. 2005. 184 f. Dissertação (Mestrado em Ciência da Informação) - Programa de Pós Graduação em Ciência da Informação, IBICT/Universidade Federal da Bahia, 2005.

OLIVEIRA, A. M.; SILVA, I. Study of information channels utilized by associated doctors of UNIMED - Ponta Grossa, Paraná-Brazil. 2005. In: CONGRESSO MUNDIAL DE INFORMAÇÃO EM SAÚDE E BIBLIOTECAS, 9., Salvador, 2005. Anais... Salvador: [s.n.], 2005. Disponível em: <http://www.icml9.org/>. Acesso em: 12 fev. 2007.

OSHEROFF, J. A . et al. Physicians' information needs: analysis of questions posed during clinical teaching. Annals of Internal Medicina, v. 114, n. 7, p. 576-581, 1991.

OSPINA, E. G.; REVEIZ HERAULT, L.; CARDONA, A. F. Uso de bases de datos bibliográficas por investigadores biomédicos latinoamericanos hispanoparlantes: estudio transversal. Revista Panamericana de Salud Pública, Washington, v. 17, n. 4, p. 230-236, Apr. 2005. Disponível em: $<$ http://www.ingentaconnect.com/content/paho/pajph;jsessionid=299c5v3x7kl9n.alice $>$.

Acesso em: 04 jun. 2008. 
RAMOS, K.; LINSCHEID, R.; SCHAFER, S. Real-time information-seeking behavior of residence physicians. Family Medicine, v. 35, n. 4, p. 257260, Apr. 2003.

RENWICK, S. Knowledge and use of electronic information resources by medical sciences faculty at the University of the West Indies. Journal of Medical Library Association, Chicago, v. 93, n.1, p. 21-31, jan. 2005. Disponível em:

<http://www.pubmedcentral.nih.gov/picrender.fcgi?artid=545116\& blobtype=pdf $>$. Acesso em: 16 fev. 2007.

SMITH, R. What clinical information do doctors need? British Medical Journal, London, v. 313, n. 7064, p. 1062-1068, Oct. 1996.

STINSON, E. R.; MUELLER, D. A. Survey of health professionals' information habits and needs: conducted through personal interviews. The Journal of the American Medical Association, Chicago, v. 243, n. 2, p. 140143, 1980.

WOOLF, S. H.; BENSON, D. A. The medical information needs of internists and pediatricians at an academic medical center. Bulletin of the Medical Library Association, Chicago, v. 77, n. 4, p. 372-380, 1989. 